\title{
Résurgence de la syphilis congénitale à Winnipeg, Manitoba
}

\author{
Peter Benoit ${ }^{1 \star}$, Lana Tennenhouse ${ }^{2}$, Alicia Lapple ${ }^{1}$, Gillian Hill-Carroll1', Souradet Shaw², \\ Jared Bullard ${ }^{2,3}$, Pierre Plourde ${ }^{1,2}$
}

\section{Résumé}

Contexte : Les taux de syphilis infectieuse ont augmenté à Winnipeg, au Manitoba, chez les personnes en âge de procréer. Une infection prénatale non traitée ou incorrectement traitée entraîne souvent une syphilis congénitale, avec des conséquences dévastatrices pour la santé et la survie du fœtus. L'objectif de cette étude était d'examiner les données de surveillance de la santé publique concernant l'incidence de la syphilis congénitale et les facteurs de risque des personnes enceintes à Winnipeg de 2018 à 2020.

Méthode : Les données extraites d'une base de données de surveillance de la population tenue par les enquêtes de santé publique de l'Office régional de la santé de Winnipeg pour tous les cas probables ou confirmés de syphilis congénitale précoce ou de mortinaissance syphilitique de 2018 à 2020 ont été examinés. Les taux de syphilis congénitale ont été calculés pour 1000 naissances vivantes. Des analyses descriptives ont été effectuées pour décrire l'âge des personnes enceintes le quartier de résidence, la consommation de substances par voie intraveineuse, l'implication des Services à l'enfance et à la famille, l'accès aux soins prénataux et l'obtention d'un traitement prénatal adéquat.

Résultats : Il y a eu huit cas de syphilis congénitale confirmée/probable en 2018, 22 cas en 2019 et 30 cas en 2020 . L'âge moyen des personnes enceintes était de 26,5 à 27,0 ans. La majorité $(66,7 \%)$ des personnes enceintes vivaient dans des quartiers du centre-ville où des éclosions de syphilis infectieuse étaient connues. Plus de $50 \%$ des personnes enceintes n'ont reçu aucun soin prénatal, ou les soins reçus consistaient en un traitement ou un suivi inadéquat. Une réinfection chez les personnes enceintes ayant reçu des soins prénataux a été suspectée dans $23,3 \%$ des cas supplémentaires.

Conclusion : Les taux de syphilis congénitale à Winnipeg ont augmenté considérablement. Les efforts de la santé publique et des prestataires de soins de santé pour répondre aux besoins de la communauté sont essentiels pour promouvoir l'accès à des soins prénataux sûrs et efficaces.
Cette oeuvre est mise à la disposition selon les termes de la licence internationale Creative Commons Attribution 4.0

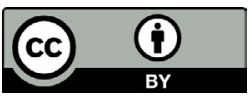

\begin{abstract}
Affiliations
${ }^{1}$ Programme de la santé de la population et du public, Office régional de la santé de Winnipeg, Winnipeg, MB

${ }^{2}$ Collège de médecine Max Rady, Faculté des sciences de la santé Rady, Université du Manitoba, Winnipeg, MB

${ }^{3}$ Laboratoire provincial Cadham, Santé et Soins aux personnes âgées du Manitoba, Winnipeg, $\mathrm{MB}$
\end{abstract}

${ }^{\star}$ Correspondance : pbenoit2@wrha.mb.ca

Citation proposée : Benoit P, Tennenhouse LG, Lapple A, Hill-Carroll G, Shaw SY, Bullard J, Plourde P. Résurgence de la syphilis congénitale à Winnipeg, Manitoba. Relevé des maladies transmissibles au Canada 2022;48(2/3):100-6. https://doi.org/10.14745/ccdr.v48i23a06f

Mots-clés : syphilis congénitale, surveillance, soins prénataux, inégalités en matière de santé

\section{Introduction}

Les taux de syphilis ont augmenté au Canada; ils ont plus que doublé entre 2016 et 2020 (1), les augmentations les plus considérables étant observées dans les provinces des Prairies. Au cours de la même période, les taux de syphilis congénitale ont été multipliés par 10 à 15, en raison de grandes éclosions de syphilis parmi la population hétérosexuelle, principalement dans les provinces des Prairies.
La syphilis congénitale se produit par la transmission verticale du spirochète Treponema pallidum d'une personne enceinte au fœtus pendant la grossesse. Les manifestations vont de l'infection asymptomatique à l'avortement spontané, la mortinaissance et l'atteinte multisystémique avec de graves conséquences pour la santé du nourrisson (2). Le risque de transmission au fœtus est élevé, avec des estimations de 70 à $100 \%$ chez les femmes enceintes non traitées atteintes de syphilis primaire ou secondaire, de $40 \%$ en cas de syphilis 
latente précoce et de moins de $10 \%$ en cas de syphilis latente tardive (3). Cependant, la syphilis congénitale est presque entièrement évitable avec un traitement prénatal adéquat, à savoir la pénicilline $\mathrm{G}$ au moins 30 jours avant I'accouchement (4). Le dépistage de la syphilis infectieuse lors du premier rendez-vous de soins prénataux est considéré comme la norme de soins au Canada. Un dépistage plus poussé peut être envisagé dans les populations à haut risque/ touchées par une éclosion (3). Le manque d'accès aux soins prénataux s'est avéré être un important facteur de risque modifiable de syphilis congénitale aux États-Unis (5). Étant donné que les conséquences de la syphilis congénitale sont importantes et qu'un dépistage et un traitement efficaces sont accessibles, l'occurrence des cas de syphilis congénitale est une mesure importante pour évaluer le succès de nos systèmes de santé publique et de soins de santé. S'il fonctionne de manière optimale, nous ne devrions pas voir de cas de syphilis congénitale.

À Winnipeg, aucun cas de syphilis congénitale n'avait été enregistré depuis 1977, et plus récemment, le premier cas détecté au Manitoba l'a été en 2015 (dans une communauté du Nord), le premier cas à Winnipeg a été détecté en 2018. Cependant, on observe depuis une augmentation considérable des cas de syphilis infectieuse, avec un taux normalisé selon I'âge de 79,5 et 140,4 cas pour 100000 individus à Winnipeg en 2018 et 2019, respectivement (données non publiées, Pierre Plourde). Cela représente une augmentation de $81 \%$ des cas en 2018 par rapport à 2017, et un nombre de cas presque doublé en 2019. Alors que la majorité des cas touchaient auparavant de manière disproportionnée les hommes ayant des rapports sexuels avec des hommes, le taux de transmission hétérosexuelle a augmenté, les femmes représentant $47 \%$ des cas de syphilis infectieuse à Winnipeg en 2018 et $54 \%$ des cas en 2019 (données non publiées, Pierre Plourde), les premiers signaux de transmission accrue chez les femmes hétérosexuelles ayant été observés dès 2015 (6). Des tendances similaires d'augmentation de l'incidence de la syphilis infectieuse chez les femmes ont été observées dans d'autres provinces canadiennes, notamment en Colombie-Britannique et en Alberta $(7,8)$. La présence d'une syphilis infectieuse non diagnostiquée ou insuffisamment traitée chez les personnes en âge de procréer représente un échec du système de santé et signifie qu'il est essentiel d'intensifier les efforts de santé publique et des prestataires de soins de santé visant à prévenir la syphilis congénitale, en collaboration avec les organismes communautaires impliqués dans la sensibilisation des personnes enceintes.

Notre objectif était d'examiner les données de surveillance de la santé publique concernant l'incidence de la syphilis congénitale à Winnipeg de 2018 à 2020, et de décrire les caractéristiques des personnes enceintes donnant naissance à des nourrissons atteints de syphilis congénitale. Les années 2018 à 2020 ont été sélectionnées comme horizon temporel, car avant 2018, aucun cas de syphilis congénitale n'avait été signalé à Winnipeg depuis plus de 40 ans.

\section{Méthode}

\section{Source des données}

Les cas déclarés de syphilis congénitale dans la base de données de surveillance de l'Office régional de la santé de Winnipeg ont été examinés de janvier 2018 à décembre 2020. Winnipeg est la plus grande ville du Manitoba, avec une population d'environ 700000 habitants en 2016 (9), 8675 naissances vivantes en 2018 et 8560 naissances vivantes en 2019, selon la base de données du programme Rôle parental et développement de la petite enfance de l'Office régional de la santé de Winnipeg (communication personnelle, Dr Christopher Green, Université du Manitoba).

Tous les cas de syphilis infectieuse et congénitale doivent être signalés à Santé publique au niveau provincial. Les cas sont classés comme des cas de Winnipeg en utilisant le code postal de la plus récente adresse connue, ou comme des individus dont on sait qu'ils vivaient à Winnipeg pendant la durée de la grossesse sans avoir une adresse à Winnipeg dans le dossier. Les cas sont gérés par des infirmières de santé publique qui remplissent des formulaires d'enquête, recueillent les caractéristiques sociodémographiques de la personne et complètent le dossier de santé publique par des notes cliniques pertinentes. Pour les enquêtes sur la syphilis congénitale, les dossiers de santé publique sont conservés séparément pour la personne enceinte et le nourrisson. Ces dossiers sont conservés dans une base de données de surveillance de la population.

Les cas déclarés de syphilis congénitale possible sont classés par Santé publique Manitoba en syphilis précoce confirmée ou probable et, à partir de 2019, comprennent également les classifications de mortinatalité syphilitique confirmée ou probable. Sur la base des définitions de cas fournies par le ministère de la Santé et Soins aux personnes âgées du Manitoba (voir l'annexe), la classification des cas est établie par l'apport conjoint d'une infirmière en santé publique et d'un spécialiste des maladies infectieuses pédiatriques, suivi de l'examen d'un médecin hygiéniste.

\section{Mesures}

Les données de santé publique provenant des enquêtes sur les cas pour la personne enceinte et le nourrisson ont été examinées pour tous les cas confirmés ou probables de syphilis congénitale à Winnipeg entre janvier 2018 et décembre 2020. Au moins deux personnes ont examiné chaque dossier indépendamment. Des données sur les caractéristiques suivantes des personnes enceintes ont été recueillies : 1) âge (années), 2) quartier de résidence (Winnipeg Centre-ville, Point Douglas ou autre), 3) consommation de substances par voie intraveineuse au cours de l'année écoulée (oui, non), 4) intervention des Services à l'enfance et à la famille (oui, non), 5) traitement prénatal adéquat défini comme la réception d'au moins un traitement de pénicilline G 2,4 millions d'unités au moins 30 jours avant l'accouchement (oui, non ou inconnu), et 6) réception de soins prénataux (aucun, certains, mais n'ont pas reçu de traitement 
adéquat, certain, mais risque élevé de nouvelle exposition ou de réinfection au troisième trimestre non traité adéquatement, certains et traités adéquatement, ou inconnu).

\section{Analyse}

Les moyennes, médianes, écarts interquartiles, fréquences et pourcentages ont été utilisés pour décrire les caractéristiques des personnes enceintes de la population étudiée. Le taux de naissance de nourrissons atteints de syphilis congénitale pour 1000 naissances vivantes a été calculé pour chaque année de la période de surveillance, en utilisant comme dénominateur le nombre total estimé de naissances vivantes par an à Winnipeg (estimé à 8600 pour 2020).

\section{Éthique}

L'approbation de l'éthique de la recherche n'était pas requise pour l'analyse et le rapport des données de surveillance de la santé publique collectées couramment.

\section{Résultats}

On a recensé 60 cas de syphilis congénitale confirmée ou probable à Winnipeg de 2018 à 2020 (tableau 1, figure 1). Huit de ces cas sont survenus en 2018, 22 en 2019 et 30 en 2020. Cela correspond à des taux de syphilis congénitale (pour 1000 naissances vivantes) de 0,9 en 2018, 2,6 en 2019 et 3,5 en 2020.

Tableau 1 : Cas incidents de syphilis congénitale et facteurs de risque à Winnipeg, au Manitoba, de 2018 à 2020

\begin{tabular}{|c|c|c|c|c|c|c|}
\hline \multirow[t]{2}{*}{ Caractéristique } & \multicolumn{2}{|c|}{$\begin{array}{c}2018 \\
(N=8)\end{array}$} & \multicolumn{2}{|c|}{$\begin{array}{c}2019 \\
(N=22)\end{array}$} & \multicolumn{2}{|c|}{$\begin{array}{c}2020 \\
(N=30)\end{array}$} \\
\hline & $\mathbf{n}$ & $\%$ & $\mathbf{n}$ & $\%$ & $\mathbf{n}$ & $\%$ \\
\hline \multicolumn{7}{|c|}{ Issues indésirables de la grossesse } \\
\hline $\begin{array}{l}\text { Syphilis } \\
\text { congénitale } \\
\text { précoce confirmée }\end{array}$ & 5 & 62,5 & 13 & 59,1 & 13 & 43,3 \\
\hline $\begin{array}{l}\text { Syphilis } \\
\text { congénitale } \\
\text { précoce probable }\end{array}$ & 3 & 37,5 & 5 & 22,7 & 9 & 30,0 \\
\hline $\begin{array}{l}\text { Mortinaissance } \\
\text { syphilitique } \\
\text { confirmée en } \\
\text { laboratoire }\end{array}$ & s.o. & s.o. & 1 & 4,5 & 6 & 20,0 \\
\hline $\begin{array}{l}\text { Probable } \\
\text { mortinaissance } \\
\text { syphilitique }\end{array}$ & s.o. & s.o. & 3 & 13,6 & 2 & 6,7 \\
\hline $\begin{array}{l}\text { Âge de la personne } \\
\text { enceinte (années) } \\
\text { au moment de } \\
\text { l'accouchement, } \\
\text { moyenne/médiane } \\
\text { (écart interquartile) }\end{array}$ & $26,6 / 27$ & $23-30$ & $27,0 / 26$ & $22-30$ & $26,5 / 25$ & $22-31$ \\
\hline \multicolumn{7}{|c|}{ Quartier de résidence } \\
\hline Centre-ville & 3 & 37,5 & 10 & 45,5 & 10 & 33,3 \\
\hline Point Douglas & 3 & 37,5 & 9 & 40,9 & 5 & 16,7 \\
\hline Autre & 2 & 25,0 & 3 & 13,6 & 15 & 50,0 \\
\hline
\end{tabular}

Tableau 1 : Cas incidents de syphilis congénitale et facteurs de risque à Winnipeg, au Manitoba, de 2018 à 2020 (suite)

\begin{tabular}{|c|c|c|c|c|c|c|}
\hline \multirow{2}{*}{ Caractéristique } & \multicolumn{2}{|c|}{$\begin{array}{c}2018 \\
(N=8)\end{array}$} & \multicolumn{2}{|c|}{$\begin{array}{c}2019 \\
(N=22)\end{array}$} & \multicolumn{2}{|c|}{$\begin{array}{c}2020 \\
(N=30)\end{array}$} \\
\hline & $\mathbf{n}$ & $\%$ & $\mathbf{n}$ & $\%$ & $\mathbf{n}$ & $\%$ \\
\hline \multicolumn{7}{|l|}{ Facteurs de risque } \\
\hline $\begin{array}{l}\text { Consommation } \\
\text { de substances par } \\
\text { voie intraveineuse } \\
\text { au cours de l'année } \\
\text { écoulée }\end{array}$ & 4 & 50,0 & 9 & 40,9 & 3 & 10,0 \\
\hline $\begin{array}{l}\text { Participation des } \\
\text { Services à l'enfance } \\
\text { et à la famille }\end{array}$ & 4 & 50,0 & 8 & 36,4 & 4 & 13,3 \\
\hline \multicolumn{7}{|c|}{ Traitement adéquat 30 jours ou plus avant l'accouchement } \\
\hline Oui & 0 & 0,0 & 6 & 27,3 & 2 & 6,7 \\
\hline Non & 8 & 100,0 & 14 & 63,6 & 25 & 83,3 \\
\hline Inconnu & 0 & 0,0 & 2 & 9,1 & 3 & 10,0 \\
\hline \multicolumn{7}{|l|}{ Soins prénataux } \\
\hline Aucun & 3 & 37,5 & 7 & 31,8 & 14 & 46,7 \\
\hline $\begin{array}{l}\text { Certains, mais } \\
\text { n'ont pas reçu de } \\
\text { traitement adéquat }\end{array}$ & 3 & 37,5 & 3 & 13,6 & 3 & 10,0 \\
\hline $\begin{array}{l}\text { Quelques-uns, } \\
\text { mais le risque de } \\
\text { nouvelle exposition } \\
\text { ou de réinfection au } \\
\text { troisième trimestre } \\
\text { n'est pas traité de } \\
\text { manière adéquate }\end{array}$ & 2 & 25,0 & 4 & 18,2 & 8 & 26,7 \\
\hline $\begin{array}{l}\text { Certains, traités de } \\
\text { manière adéquate }\end{array}$ & 0 & 0,0 & 6 & 27,3 & 2 & 6,7 \\
\hline Inconnu & 0 & 0,0 & 2 & 9,1 & 3 & 10,0 \\
\hline
\end{tabular}

Figure 1 : Syphilis congénitale précoce à Winnipeg, au Manitoba, selon le mois/année de naissance, de 2018 à 2020

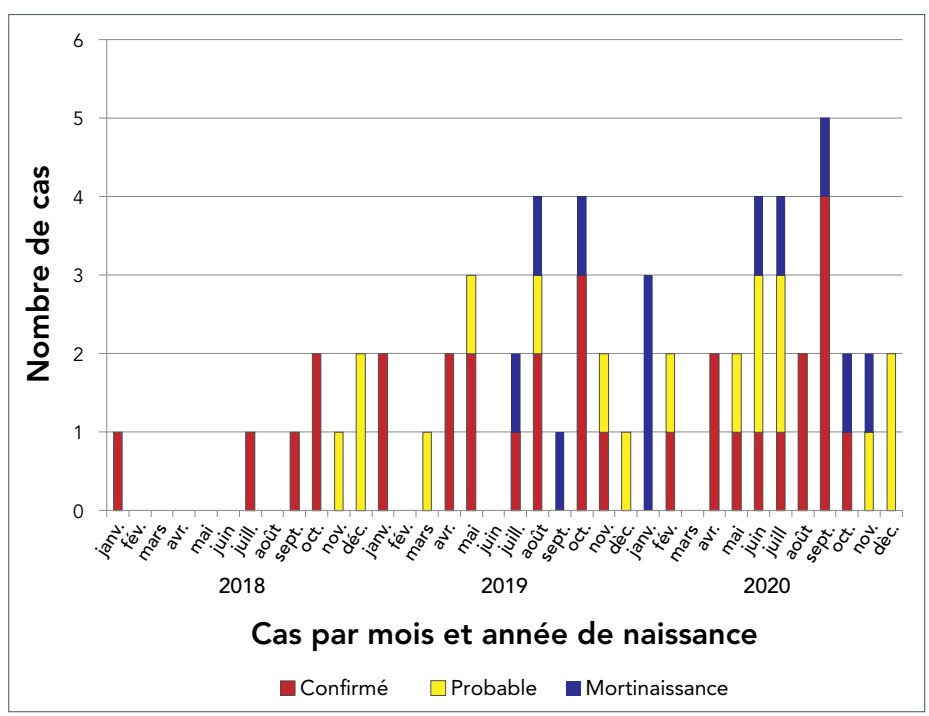


L'âge médian de la personne enceinte se situait entre 26,5 et 27,0 ans pour chaque année civile tout au long de la période de surveillance. La majorité des personnes enceintes vivaient dans deux quartiers particuliers de Winnipeg (centre-ville et Point Douglas), tous deux considérés comme faisant partie du noyau central de la ville et historiquement associés à des éclosions $d$ 'infections sexuellement transmissibles $(5,10)$. Ces quartiers du centre-ville sont connus pour avoir des taux plus élevés de pauvreté, de populations racialisées et de criminalité. Ils sont également bien étudiés en termes de données sur la mortalité et sont connus pour présenter une espérance de vie nettement inférieure à celle du reste de Winnipeg. La consommation de substances par voie intraveineuse au cours de l'année écoulée a été signalée chez $50,0 \%$ des personnes enceintes en 2018 , 40,9\% en 2019 et 10,0\% en 2020. Les Services à l'enfance et à la famille ont été impliqués dans $50,0 \%$ des cas en $2018,36,4 \%$ en 2019 et $13,3 \%$ en 2020 . Sur les 16 personnes enceintes qui ont déclaré avoir consommé des substances par voie intraveineuse, 11 (69\%) ont mentionné la méthamphétamine en cristaux.

Plus de $50 \%$ des personnes enceintes n'ont reçu aucun soin prénatal ou n'ont reçu qu'une quantité limitée de soins prénataux (généralement une seule visite) avec un suivi et un traitement inadéquats. En moyenne de 2018 à 2020, 23,3 \% des personnes enceintes ont reçu des soins prénataux; cependant, une réinfection ou une nouvelle exposition à haut risque a été suspectée au troisième trimestre. Aucun des personnes enceintes n'a reçu de traitement adéquat pendant la grossesse en 2018, bien que ce chiffre ait augmenté pour atteindre 27,3 \% en 2019, avant de diminuer à nouveau à 6,7 \% en 2020.

\section{Discussion}

En 2007, l'Organisation mondiale de la Santé a publié l'objectif de réduire les taux de syphilis congénitale à maximum 0,5 cas pour 1000 naissances vivantes (11). Avec une augmentation des cas de syphilis congénitale chaque année et un taux de 3,5 cas pour 1000 naissances vivantes en 2020, Winnipeg est loin d'atteindre cet objectif. Le jeune âge à l'accouchement, le fait de résider dans certains quartiers du centre-ville présentant d'importantes éclosions de syphilis infectieuse, la consommation de drogues par voie intraveineuse et l'intervention des Services à l'enfance et à la famille sont apparus comme des cofacteurs possibles pour les personnes enceintes dans cette population. Cela suggère fortement que la pauvreté et les désavantages sociaux structurels jouent un rôle important dans l'apparition de la syphilis congénitale.

Le manque d'accès à des soins prénataux adéquats pendant la grossesse était présent chez plus de $50 \%$ des personnes évaluées pendant la période de surveillance. Les iniquités dans l'accès aux soins prénataux sont un problème connu à Winnipeg, les recherches indiquant que 15 à $21 \%$ des femmes enceintes résidant dans le noyau central de Winnipeg ont reçu des soins prénataux inadéquats de 2004-2005 à 2008-2009 (12). Les personnes les plus vulnérables peuvent avoir des difficultés à accéder au système de soins de santé pour diverses raisons, notamment le manque de moyens de transport, les difficultés linguistiques, la crainte des préjugés des prestataires de soins de santé découlant du racisme et le manque de connaissances sur le système de soins de santé (13). Par conséquent, les approches efficaces de la gestion des taux croissants de syphilis congénitale doivent tenir compte des besoins uniques de la communauté. En Alberta, un programme a été mis en œuvre pour fournir des soins prénataux aux personnes vivant dans la rue, dans le but de déterminer la faisabilité de la décolonisation des soins prénataux par la sensibilisation des membres de la communauté du centre-ville (14). Les participants à l'étude ont fait état d'un taux de satisfaction élevé à l'égard du programme, et ont particulièrement insisté sur le fait qu'ils appréciaient que les prestataires de soins les soutiennent et ne les jugent pas. À Winnipeg, des efforts de collaboration entre la santé publique et les organisations communautaires autochtones sont en cours dans le but d'établir des approches dirigées par les Autochtones pour la gestion des infections transmissibles sexuellement et par le sang; toutefois, les difficultés à obtenir du financement pour les stratégies de lutte contre les infections sexuellement transmissibles et transmissibles par le sang dirigées par les Autochtones demeurent un défi important.

Un sous-ensemble de la population qui a bénéficié de soins prénataux a été soupçonné d'avoir été exposé à nouveau à la syphilis infectieuse quelque temps après son premier dépistage prénatal négatif. Plusieurs personnes ont été traitées avec une réduction documentée de la réponse sérologique pendant la grossesse, suivie de preuves sérologiques de réinfection (i.e. une augmentation des titres après une diminution). II est également possible que certaines personnes présentant une sérologie syphilis négative lors de la première visite prénatale aient déjà développé une syphilis infectieuse au moment du dépistage initial, mais que leur infection soit trop précoce pour avoir développé une réponse immunitaire détectable. Un rapport de la ville de New York indique qu'environ un tiers de ses cas de syphilis congénitale sont survenus au cours de la grossesse de femmes dont le dépistage initial était négatif (15). Ces résultats soulignent l'importance du dépistage répété de la syphilis infectieuse pendant la grossesse chez les femmes vivant dans des communautés où des éclosions sont connues et qui présentent d'autres facteurs de risque. Compte tenu de l'augmentation des taux de syphilis congénitale, depuis le début de 2019, il a été conseillé aux prestataires de soins de santé de Winnipeg de tester toutes les femmes enceintes pour la syphilis infectieuse à la $28^{\mathrm{e}}$ semaine de grossesse et à l'accouchement, en plus du dépistage de routine lors de la première visite prénatale.

Les données relatives à l'origine ethnique n'ont pas été recueillies de manière systématique au cours de la période de surveillance. Cela représente une importante occasion manquée. On a constaté que les groupes racialisés supportent de manière disproportionnée le fardeau de la syphilis congénitale, ainsi un 
rapport indique que $85 \%$ des femmes ayant donné naissance à des enfants atteints de syphilis congénitale à Los Angeles étaient soit latino-américaines, soit afro-américaines (16). Au Canada, on sait que les autochtones ont des difficultés à accéder à des soins de santé sûrs en raison de leur expérience du racisme dans un système colonialiste (17). Cette situation souligne l'importance de recueillir systématiquement des données sur l'origine ethnique dans le cadre de la surveillance future de la syphilis congénitale, afin de garantir que les interventions et les modèles de soins ultérieurs sont fournis de manière sûre et appropriée sur le plan culturel. La collaboration avec les Autochtones et d'autres groupes racialisés serait essentielle pour garantir que ces données soient collectées et rapportées de manière respectueuse et non stigmatisante.

\section{Forces et faiblesses}

Ces données de surveillance présentaient des points forts et des limites. Les données sur l'origine ethnique n'ont pas été collectées systématiquement. Les données relatives à la consommation de substances par voie intraveineuse ont été recueillies au moyen de l'examen des dossiers de données de surveillance et reposaient sur le fait que les clients déclaraient eux-mêmes leur consommation. Par conséquent, il est possible que la consommation de substances par voie intraveineuse n'ait pas été déclarée à sa juste valeur. Certaines conséquences potentielles de la syphilis congénitale, comme l'avortement spontané, n'ont pas été prises en considération. De plus, les mortinaissances syphilitiques n'ont pas été incluses dans la base de données de surveillance en 2018 et peut-être au début de 2019, ce qui pourrait avoir entraîné une sous-déclaration de la syphilis congénitale. Enfin, les changements apportés aux formulaires d'enquête sur les cas de santé publique et à la méthodologie de saisie des données au cours de la période de surveillance peuvent avoir entraîné des incohérences possibles dans la déclaration des données sur les caractéristiques des personnes enceintes d'une année à l'autre. L'un des points forts de l'étude est l'utilisation de données fondées sur la population avec des classifications rigoureusement définies des cas de syphilis congénitale. De plus, le codirecteur actuel du laboratoire provincial de santé publique, où tous les cas de syphilis congénitale sont testés, est un spécialiste des maladies infectieuses pédiatriques, ce qui devrait garantir une précision maximale dans la classification des cas.

\section{Conclusion}

Les taux de syphilis congénitale augmentent à Winnipeg, parallèlement à l'augmentation des taux de syphilis infectieuse chez les personnes en âge de procréer. Le manque d'accès aux soins prénataux est apparu comme un facteur de risque majeur de syphilis congénitale. L'importance du dépistage répété de la syphilis infectieuse pendant la grossesse dans les populations à haut risque a également été démontrée. L'élimination des obstacles systémiques aux soins prénataux et la mise en œuvre de modèles de soins culturellement sûrs et adaptés aux besoins de la communauté peuvent constituer des interventions efficaces pour faire face à l'augmentation des taux de syphilis congénitale. Les études et interventions futures devraient mettre l'accent sur une approche de recherche communautaire, où l'implication précoce de la communauté est une priorité essentielle pour entrer en contact avec les personnes les plus à risque. Cela permettra aux chercheurs d'en savoir plus sur les facteurs clés qui augmentent le risque de syphilis congénitale et de fournir un accès aux ressources de la manière la plus accessible pour ceux qui, traditionnellement, n'ont pas accès aux ressources clés telles que les soins prénataux. Sans ces changements d'approche, les interventions colonialistes traditionnelles risquent de ne pas être efficaces pour lutter contre l'augmentation des taux de syphilis congénitale.

\section{Déclaration des auteurs}

P. B. - Analyse et interprétation des données de surveillance, rédaction de la première version du manuscrit, analyse des données et révisions du manuscrit

L. G. T. - Analyse et interprétation des données de surveillance, rédaction de la première version du manuscrit, analyse des données et révisions du manuscrit

A. L. - Enquête, expertise clinique, et révisions du manuscrit G. H. C. - Enquête, expertise clinique, et révisions du manuscrit J. B. - Enquête, expertise clinique, et révisions du manuscrit S. Y. S. - Expertise académique et méthodologique, analyse des données et révisions du manuscrit

P. P. - Conceptualisation du projet, supervision

Tous les auteurs ont contribué de manière égale à cette analyse de surveillance.

Le contenu de l'article et les points de vue qui y sont exprimés n'engagent que les auteurs et ne correspondent pas nécessairement à ceux du gouvernement du Canada.

\section{Intérêts concurrents}

Aucun.

\section{Remerciements}

Nous tenons à remercier l'équipe pour la santé sexuelle et la réduction des méfaits des infirmières en santé publique de l'Office régional de la santé de Winnipeg (ORSW), qui a travaillé avec diligence pour recueillir les données de surveillance utilisées dans le présent rapport. Pour les personnes en âge de procréer qui ne sont pas en mesure d'accéder à des soins prénataux appropriés, nous reconnaissons les répercussions importantes que cette situation a sur leur santé et celle de leurs nourrissons. Nous pensons que les principaux obstacles peuvent et doivent être éliminés et que cela devrait être une priorité essentielle pour les systèmes de santé publique et de soins de santé du Manitoba et du Canada. 


\section{Financement}

S. Y. S. est soutenu par une Chaire de recherche du Canada en science des programmes et santé publique mondiale (niveau 2).

\section{Références}

1. Agence de la santé publique du Canada. Syphilis infectieuse et syphilis congénitale au Canada, 2020 (infographie). Ottawa (ON) : ASPC; 2021. https://www.canada.ca/fr/ sante-publique/services/publications/maladies-et-affections/ syphilis-infectieuse-syphilis-congenitale-canada-2020.html

2. Arnold SR, Ford-Jones EL. Congenital syphilis: A guide to diagnosis and management. Paediatr Child Health 2000;5(8):463-9. DOI PubMed

3. Robinson JL; Canadian Paediactric Society, Infectious Diseases and Immunization Committee. Congenital syphilis: no longer just of historical interest. J Paediatr Child Health 2009;14(5):337. https://cps.ca/en/documents/position/ congenital-syphilis

4. Wendel GD Jr, Sheffield JS, Hollier LM, Hill JB, Ramsey PS, Sánchez PJ. Treatment of syphilis in pregnancy and prevention of congenital syphilis. Clin Infect Dis 2002;35 Suppl 2:S200-9. DOI PubMed

5. Kimball A, Torrone E, Miele K, Bachmann L, Thorpe $\mathrm{P}_{\text {, }}$ Weinstock H, Bowen V. Missed Opportunities for Prevention of Congenital Syphilis - United States, 2018. MMWR Morb Mortal Wkly Rep 2020;69(22):661-5. DOI PubMed

6. Shaw SY, Ross C, Nowicki DL, Marshall S, Stephen S, Davies C, Riddell J, Bailey K, Elliott LJ, Reimer JN, Plourde PJ. Infectious syphilis in women: what's old is new again? Int J STD AIDS 2017;28(1):77-87. DOI PubMed

7. Grennan T, Giesbrecht E, Ogilvie G, Pederson A, van Schalkwyk J, Gilbert M, Wong J. Syphilis outbreak in $\mathrm{BC}$ : Changes to syphilis screening in pregnancy. BCMJ. 2019;61(8):328. https://bcmj.org/bccdc/syphilis-outbreak-bcchanges-syphilis-screening-pregnancy

8. Singh A, Romanowski B. The return of syphilis in Canada: A failed plan to eliminate this infection. JAMMI 2019;4(4):215-7. DOI
9. Statistique Canada. Profil du recensement, Recensement de 2016. Winnipeg, CY [Subdivision de recensement], Manitoba et Canada [Pays] (tableau). Statistique Canada numéro 98-316-X2016001 au catalogue. Ottawa (ON) : StatCan; 2017. https://www12.statcan.gc.ca/censusrecensement/2016/dp-pd/prof/index.cfm?Lang=F

10. Elliott LJ, Blanchard JF, Beaudoin CM, Green CG, Nowicki DL, Matusko P, Moses S. Geographical variations in the epidemiology of bacterial sexually transmitted infections in Manitoba, Canada. Sex Transm Infect 2002;78 Suppl 1:i139-44. DOI PubMed

11. Organisation mondiale de la Santé. L'élimination mondiale de la syphilis congénitale : raison d'être et stratégie. Genève (Suisse) : OMS; 2007. https://www.who.int/ reproductivehealth/publications/rtis/9789241595858/fr/

12. Heaman MI, Martens PJ, Brownell MD, Chartier MJ, Thiessen KR, Derksen SA, Helewa ME. Inequities in utilization of prenatal care: a population-based study in the Canadian province of Manitoba. BMC Pregnancy Childbirth 2018;18(1):430. DOI PubMed

13. Loignon C, Hudon C, Goulet É, Boyer S, De Laat M, Fournier N, Grabovschi C, Bush P. Perceived barriers to healthcare for persons living in poverty in Quebec, Canada: the EQUIhealThY project. Int J Equity Health 2015;14(1):4. DOl PubMed

14. Mill J, Singh A, Taylor M. Women in the Shadows. Can J Urban Res 2012;21(2):68-89. https://www.jstor.org/ stable/26193913

15. Slutsker JS, Hennessy RR, Schillinger JA. Factors Contributing to Congenital Syphilis Cases - New York City, 2010-2016. MMWR Morb Mortal Wkly Rep 2018;67(39):1088-93. DOI PubMed

16. Los Angeles County Department of Public Health Division of HIV and STD Programs. Eliminating congenital syphilis in Los Angeles County: A call to action. Los Angeles (CA): LA County Public Health; 2020. http://publichealth.lacounty. gov/dhsp/Providers/CS_EliminationPlanDraft_01282020.pdf

17. Allen L, Hatala A, ljaz S, Courchene ED, Bushie EB. Indigenous-led health care partnerships in Canada. CMAJ 2020;192(9):E208-16. DOl PubMed 


\section{Annexe : Définitions de cas de syphilis congénitale du ministère de la Santé et des Soins aux personnes âgées du Manitoba}

\section{Cas de syphilis congénitale précoce confirmé en laboratoire (dans les deux ans suivant la naissance)}

- Identification de T. pallidum par microscopie sur fond noir, anticorps à fluorescence directe, ou détection de I'ADN de T. pallidum par un test d'amplification des acides nucléiques (e.g. réaction en chaîne par polymérase; PCR) dans un échantillon clinique approprié, ou examen équivalent de matériel provenant d'écoulements nasaux, de lésions cutanées, du placenta ou du cordon ombilical, ou de matériel d'autopsie d'un nouveau-né (jusqu'à l'âge de 4 semaines)

OU

- Sérologie réactive (tréponémique et non tréponémique) à partir de sang veineux (pas de sang de cordon ombilical) chez un nourrisson/enfant présentant ou non des signes cliniques, de laboratoire ou radiographiques compatibles avec une syphilis congénitale*, mais présentant l'un ou les deux éléments suivants :

- Augmentation des titres sérologiques de la syphilis lors du suivi lorsqu'il existe des éléments probants indiquant que la mère a eu une infection syphilitique pendant la grossesse

- Titres supérieurs ou égaux à quatre fois ceux de la mère lorsqu'ils sont prélevés en même temps ou dans la semaine qui suit, dans la période postnatale immédiate

$\mathrm{OU}$

- Sérologie réactive (tréponémique et non tréponémique) à partir de sang veineux (pas de sang de cordon ombilical) chez un nourrisson/enfant présentant des signes cliniques, de laboratoire ou radiographiques compatibles avec une syphilis congénitale*, dont la mère était séropositive ou présentait un PCR positif pour la syphilis au cours de la OU grossesse ou lors de l'accouchement

- Un enfant qui ne répond pas aux critères ci-dessus, mais qui présente une sérologie tréponémique réactive persistante entre l'âge de 18 et 24 mois (indépendamment de l'état du traitement et du statut infectieux de la mère)

* Comprend tous les éléments probants de la syphilis congénitale lors de l'examen physique (e.g. hépatosplénomégalie, éruption cutanée constante, condylome lata, rhinites, pseudoparalysie), preuve de syphilis congénitale sur les radiographies des os longs, test de laboratoire de recherche sur les maladies vénériennes dans le liquide céphalorachidien réactif, numération cellulaire ou protéine élevée dans le liquide céphalorachidien sans autre cause.

\section{Cas de syphilis congénitale précoce probable (dans les deux ans suivant la naissance) ${ }^{\dagger}$}

- Sérologie réactive (tréponémique et non tréponémique) à partir de sang veineux (pas de sang de cordon ombilical) chez un nourrisson/enfant sans manifestations cliniques, de laboratoire ou radiographiques de syphilis congénitale dont la mère présentait :

- Une syphilis non traitée ou insuffisamment ${ }^{\ddagger}$ traitée à l'accouchement

OU

- Des preuves d'une réinfection ou d'une rechute au cours de la grossesse après un traitement approprié (e.g. augmentation des titres non tréponémiques au moins quatre fois plus élevés)

\begin{abstract}
† Une réaction sérologique tréponémique persistante à l'âge de 18 à 24 mois confirme le diagnostic de syphilis congénitale. Une absence de réaction sérologique (tests tréponémiques et non tréponémiques) à l'âge de 18 à 24 mois ou avant exclut le cas (i.e. qu'il ne s'agit plus d'un cas probable).

₹ Le traitement inadéquat consiste en toute thérapie sans pénicilline ou de pénicilline administrée pendant la grossesse, mais moins de 30 jours avant l'accouchement, ou malgré le traitement, on a constaté une baisse inadéquate des titres non tréponémiques. Remarque : le type de pénicilline administré est important; il s'agit généralement de pénicilline benzathine pendant la grossesse, à l'exception du traitement de la neurosyphilis.
\end{abstract}

\section{Cas confirmé en laboratoire de mortinaissance syphilitique}

- Une mort fœtale survenant après 20 semaines de grossesse avec confirmation en laboratoire de l'infection (i.e. détection de l'ADN de T. pallidum dans un échantillon clinique approprié, anticorps à fluorescence directe ou examen équivalent de matériel provenant du placenta, du cordon ombilical ou de matériel d'autopsie).

\section{Cas probable de mortinaissance syphilitique}

- Mort fœtale survenant après 20 semaines de grossesse où la mère avait une syphilis non traitée ou insuffisamment traitée avant l'accouchement OU présentait des signes de réinfection ou de rechute pendant la grossesse après un traitement approprié (tel qu'une augmentation des titres), sans qu'aucune autre cause de mortinatalité ne soit établie.

Source : https://www.gov.mb.ca/health/publichealth/cdc/ protocol/syphilis.pdf 